\title{
Harmonic function for which the second dilatation is $\alpha$-spiral
}

Melike Aydog̃an ${ }^{1 *}$, Emel Yavuz Duman², Yaşar Polatog̃lu² and Yasemin Kahramaner ${ }^{3}$

"Correspondence:

melike.aydogan@isikun.edu.tr

${ }^{1}$ Department of Mathematics, Işık

University, Meşrutiyet Koyu, Şile,

istanbul, Turkey

Full list of author information is

available at the end of the article

\begin{abstract}
Let $f=h+\bar{g}$ be a harmonic function in the unit disc $\mathbb{D}$. We will give some properties of $f$ under the condition the second dilatation is $\alpha$-spiral.
\end{abstract}

MSC: $30 C 45 ; 30 C 55$

Keywords: Harmonic functions; growth theorem; distortion theorem; coefficient inequality

\section{Introduction}

A planar harmonic mapping in the unit disc $\mathbb{D}=\{z \in \mathbb{C}|| z \mid<1\}$ is a complex-valued harmonic function $f$ which maps $\mathbb{D}$ onto some planar domain $f(\mathbb{D})$. Since $\mathbb{D}$ is simply connected, the mapping $f$ has a canonical decomposition $f=h+\bar{g}$, where $h$ and $g$ are analytic in $\mathbb{D}$. As usual, we call $h$ the analytic part of $f$ and $g$ the co-analytic part of $f$. An elegant and complete account of the theory of planar harmonic mapping is given in Duren's monograph [1].

Lewy [2] proved in 1936 that the harmonic function $f$ is locally univalent in a simply connected domain $\mathbb{D}_{1}$ if and only if its Jacobian

$$
J_{f}(z)=\left|h^{\prime}(z)\right|^{2}-\left|g^{\prime}(z)\right|^{2}>0
$$

is different from zero in $\mathbb{D}_{1}$. In view of this result, locally univalent harmonic mappings in the unit disc are either sense-reversing if

$$
\left|g^{\prime}(z)\right|>\left|h^{\prime}(z)\right|
$$

in $\mathbb{D}_{1}$ or sense-preserving if

$$
\left|g^{\prime}(z)\right|<\left|h^{\prime}(z)\right|
$$

in $\mathbb{D}_{1}$. Throughout this paper, we will restrict ourselves to the study of sense-preserving harmonic mappings. However, since $f$ is sense-preserving if and only if $\bar{f}$ is sensereserving, all the results obtained in this article regarding sense-preserving harmonic mappings can be adapted to sense-reversing ones. Note that $f=h+\bar{g}$ is sense-preserving in $\mathbb{D}$ if and only if $h^{\prime}(z)$ does not vanish in the unit disc and the second-complex dilatation $w(z)=\frac{g^{\prime}(z)}{h^{\prime}(z)}$ has the property $|w(z)|<1$ in $\mathbb{D}$; therefore, we can take $h(z)=z+a_{2} z^{2}+\cdots$, 
$g(z)=b_{1} z+b_{2} z^{2}+\cdots$. Thus, the class of all harmonic mappings being sense-preserving in the unit disc can be defined by

$$
\begin{aligned}
S_{H}= & \left\{f=h(z)+\overline{g(z)} \mid h(z)=z+a_{2} z^{2}+\cdots,\right. \\
& \left.g(z)=b_{1} z+b_{2} z^{2}+\cdots, f \text { sense-preserving }\right\} .
\end{aligned}
$$

Let $\Omega$ be the family of functions $\phi(z)$ which are regular in $\mathbb{D}$ and satisfy the conditions $\phi(0)=0,|\phi(z)|<1$ for all $z \in \mathbb{D}$. Denote by $P$ the family of functions $p(z)=1+p_{1} z+p_{2} z^{2}+$ $\cdots$ which are regular in $\mathbb{D}$ such that

$$
p(z)=\frac{1+\phi(z)}{1-\phi(z)}
$$

for some function $\phi(z) \in \Omega$ for all $z \in \mathbb{D}$.

Next, let $S^{*}$ denote the family of functions $s(z)=z+c_{2} z^{2}+c_{3} z^{3}+\cdots$ which are regular in $\mathbb{D}$ such that

$$
z \frac{s^{\prime}(z)}{s(z)}=p(z)
$$

for some $p(z) \in P$ for all $z \in \mathbb{D}$.

Let $s_{1}(z)=z+\alpha_{2} z^{2}+\alpha_{3} z_{3}+\cdots$ and $s_{2}(z)=z+\beta_{2} z^{2}+\beta_{3} z^{3}+\cdots$ be analytic functions in $\mathbb{D}$. If there exists $\phi(z) \in \Omega$ such that $s_{1}(z)=s_{2}(\phi(z))$ for all $z \in \mathbb{D}$, then we say that $s_{1}(z)$ is subordinate to $s_{2}(z)$ and we write $s_{1}(z) \prec s_{2}(z)$, then $s_{1}(\mathbb{D}) \subset s_{2}(\mathbb{D})$.

Now, we consider the following class of harmonic mappings in the plane:

$$
\begin{aligned}
S_{\mathrm{HPST}}^{*}(\alpha)= & \left\{f=h(z)+\overline{g(z)} \mid f \in S_{H}, h(z) \in S^{*},\right. \\
& \left.\operatorname{Re}\left(e^{i \alpha} w(z)\right)=\operatorname{Re}\left(e^{i \alpha} \frac{g^{\prime}(z)}{h^{\prime}(z)}\right)>0,|\alpha|<\frac{\pi}{2}\right\} .
\end{aligned}
$$

In the present paper, we will investigate the class $S_{\mathrm{HPST}}^{*}(\alpha)$.

We will need the following lemma and theorem in the sequel.

Theorem $1.1([3,4])$ Let $h(z)$ be an element of $S^{*}$, then

$$
\frac{r}{(1+r)^{2}} \leq|h(z)| \leq \frac{r}{(1-r)^{2}}
$$

for all $|z|=r<1$.

$$
\frac{1-r}{(1+r)^{3}} \leq\left|h^{\prime}(z)\right| \leq \frac{1+r}{(1-r)^{3}} .
$$

These inequalities are sharp because the extremal function is $h(z)=\frac{z}{(1-z)^{2}}$.

Lemma 1.2 ([2,5]) Let $h(z)$ and $g(z)$ be regular in $\mathbb{D}, h(z)$ map $|z|<1$ onto a many-sheeted starlike region, $\operatorname{Re}\left(e^{i \alpha} \frac{g^{\prime}(z)}{h^{\prime}(z)}\right)>0,|\alpha|<\frac{\pi}{2}$ for $|z|<1 . h(0)=g(0)=0$. Then $\operatorname{Re}\left(e^{i \alpha} \frac{g(z)}{h(z)}\right)>0$ for $|z|<1$. 


\section{Main results}

Lemma 2.1 Let $f=h(z)+\overline{g(z)}$ be an element of $S_{\mathrm{HPST}}^{*}(\alpha)$ then

$$
\frac{\left|b_{1}\right|-r}{1-\left|b_{1}\right| r} \leq\left|\frac{g^{\prime}(z)}{h^{\prime}(z)}\right| \leq \frac{\left|b_{1}\right|+r}{1+\left|b_{1}\right| r}
$$

for all $|z|=r<1$. This inequality is sharp because the extremal function is

$$
e^{i \alpha} \frac{g^{\prime}(z)}{h^{\prime}(z)}=\frac{z+b}{1+\bar{b} z}
$$

where $b=e^{i \alpha} b_{1}$.

Proof Since

$$
\begin{aligned}
& w(z)=\frac{g^{\prime}(z)}{h^{\prime}(z)}=\frac{\left(b_{1} z+b_{2} z^{2}+\cdots\right)^{\prime}}{\left(z+a_{2} z^{2}+\cdots\right)^{\prime}}=\frac{b_{1}+2 b_{2} z+\cdots}{1+2 a_{2} z+\cdots}, \\
& W(z)=e^{i \alpha} w(z)=e^{i \alpha} \frac{g^{\prime}(z)}{h^{\prime}(z)}=\frac{e^{i \alpha} b_{1}+2 e^{i \alpha} b_{2} z+\cdots}{1+2 a_{2} z+\cdots} \Rightarrow W(0)=e^{i \alpha} b_{1}=b, \\
& |W(z)|=\left|e^{i \alpha} w(z)\right|=\left|e^{i \alpha}\right||w(z)|=|w(z)|<1,
\end{aligned}
$$

then the function

$$
\phi(z)=\frac{W(z)-W(0)}{1-\overline{W(0)} W(z)}=\frac{W(0)-b}{1-\bar{b} W(0)}=\frac{b-b}{1-b^{2}}=0
$$

satisfies the condition of the Schwarz lemma. Using the definition of subordination, we have

$$
W(z)=e^{i \alpha} w(z)=e^{i \alpha} \frac{g^{\prime}(z)}{h^{\prime}(z)}=\frac{b+\phi(z)}{1+\bar{b} \phi(z)} \quad \Leftrightarrow \quad e^{i \alpha} \frac{g^{\prime}(z)}{h^{\prime}(z)} \prec \frac{b+z}{1+\bar{b} z} .
$$

On the other hand, the transformation $\left(\frac{b+z}{1+\bar{b} z}\right)$ maps $|z|<1$ onto the disc with the center

$$
C(r)=\left(\frac{\alpha_{1}\left(1-r^{2}\right)}{1-\left|b_{1}\right|^{2} r^{2}}, \frac{\alpha_{2}\left(1-r^{2}\right)}{1-\left|b_{1}\right|^{2} r^{2}}\right), \quad b=\alpha_{1}+i \alpha_{2}
$$

and the radius

$$
\rho(r)=\frac{\left(1-\left|b_{1}\right|^{2}\right) r}{1-\left|b_{1}\right|^{2} r^{2}} .
$$

Therefore, we can write

$$
\left|e^{i \alpha} \frac{g^{\prime}(z)}{h^{\prime}(z)}-\frac{b_{1}\left(1-r^{2}\right)}{1-\left|b_{1}\right|^{2} r^{2}}\right| \leq \frac{\left(1-\left|b_{1}\right|^{2}\right) r}{1-\left|b_{1}\right|^{2} r^{2}}
$$

which gives (2.1). 
Corollary 2.2 Let $f \in S_{\mathrm{HPST}}^{*}(\alpha)$, then

$$
\begin{aligned}
& \frac{r\left(\left|b_{1}\right|-r\right)}{(1+r)^{2}\left(1-\left|b_{1}\right| r\right)} \leq|g(z)| \leq \frac{r\left(\left|b_{1}\right|+r\right)}{(1-r)^{2}\left(1+\left|b_{1}\right| r\right)}, \\
& \frac{(1-r)\left(\left|b_{1}\right|-r\right)}{(1+r)^{3}\left(1-\left|b_{1}\right| r\right)} \leq\left|g^{\prime}(z)\right| \leq \frac{(1+r)\left(\left|b_{1}\right|+r\right)}{(1-r)^{3}\left(1+\left|b_{1}\right| r\right)}
\end{aligned}
$$

for all $|z|=r<1$.

Proof Using Lemma 1.2 and Lemma 2.1, then we can write

$$
\begin{aligned}
& |h(z)| \frac{\left|b_{1}\right|-r}{1-\left|b_{1}\right| r} \leq|g(z)| \leq|h(z)| \frac{\left|b_{1}\right|+r}{1+\left|b_{1}\right| r}, \\
& \left|h^{\prime}(z)\right| \frac{\left|b_{1}\right|-r}{1-\left|b_{1}\right| r} \leq\left|g^{\prime}(z)\right| \leq\left|h^{\prime}(z)\right| \frac{\left|b_{1}\right|+r}{1+\left|b_{1}\right| r} .
\end{aligned}
$$

If we use Theorem 1.1 in the inequalities (2.5) and (2.6), we get (2.3) and (2.4).

Corollary 2.3 Let $f=h(z)+\overline{g(z)}$ be an element of $S_{H P T S}^{*}(\alpha)$, then

$$
\frac{\left(1-\left|b_{1}\right|^{2}\right)(1-r)^{3}}{(1+r)^{5}\left(1+\left|b_{1}\right| r\right)^{2}} \leq J_{f(z)} \leq \frac{\left(1-\left|b_{1}\right|^{2}\right)(1+r)^{3}}{(1-r)^{5}\left(1+\left|b_{1}\right| r\right)^{2}}
$$

for all $|z|=r<1$.

Proof Since

$$
J_{f(z)}=\left|h^{\prime}(z)\right|^{2}-\left|g^{\prime}(z)\right|^{2}=\left|h^{\prime}(z)\right|^{2}\left(1-|w(z)|^{2}\right),
$$

using Lemma 2.1 and Theorem 1.1 in the equality (2.8) and after simple calculations, we get (2.7).

Corollary 2.4 If $=h(z)+\overline{g(z)}$ is an element of $S_{H P T S}^{\prime \prime}(\alpha)$, then

$$
\begin{aligned}
& \frac{1}{(1+a)^{3}(-1+r)^{2}}\left(2(1+a)(a(-2+r)-r) r+(-1+a)^{2}(-1+r)^{2} \log (1-r)\right. \\
& \left.-(-1+a)^{2}(-1+r)^{2} \log (1+a r)\right) \leq|f(z)| \leq \frac{1}{(1+a)^{2}\left(-1+r^{2}\right)^{2}} \\
& \left(-2 r\left(-1+r+4 a r+r^{2}+r^{3}-a^{2}\left(-1+r\left(-3+r+r^{2}\right)\right)\right)\right. \\
& \left.\quad+(-1+a)^{2}\left(-1+r^{2}\right)^{2} \log (1-r)-(-1+a)^{2}\left(-1+r^{2}\right)^{2} \log (1+a r)\right),
\end{aligned}
$$

where $a=\left|b_{1}\right|$ for all $|z|=r<1$.

Proof Using Corollary 2.2 and Theorem 1.1, we obtain

$$
\left(\left|h^{\prime}(z)\right|-\left|g^{\prime}(z)\right|\right) \geq \frac{\left(1-r^{4}\right)\left(1+\left|b_{1}\right| r\right)-\left(1+r^{4}\right)\left(\left|b_{1}\right|+r\right)}{(1-r)^{3}(1+r)^{3}\left(1+\left|b_{1}\right| r\right)},
$$


and

$$
\left(\left|h^{\prime}(z)\right|+\left|g^{\prime}(z)\right|\right) \leq \frac{(1+r)^{2}\left(1+\left|b_{1}\right|\right)}{(1-r)^{3}\left(1+\left|b_{1}\right| r\right)}
$$

Therefore, we have

$$
\begin{aligned}
& \left(\left|h^{\prime}(z)\right|-\left|g^{\prime}(z)\right|\right)|d z| \leq|d f| \leq\left(\left|h^{\prime}(z)\right|+\left|g^{\prime}(z)\right|\right)|d z| \\
& \quad \Rightarrow \quad \frac{(1-r)^{4}\left(1+\left|b_{1}\right| r\right)-(1+r)^{4}\left(\left|b_{1}\right|+r\right)}{(1-r)^{3}(1+r)^{3}\left(1+\left|b_{1}\right| r\right)} d r \leq|d f| \leq \frac{(1+r)^{2}\left(1+\left|b_{1}\right|\right)}{(1-r)^{3}\left(1+\left|b_{1}\right| r\right)} d r .
\end{aligned}
$$

Integrating the last inequality (2.10), we get (2.9).

Theorem 2.5 Let $f=h(z)+\overline{g(z)}$ be an element of $S_{H P T S}^{*}(\alpha)$, then

$$
\sum_{k=1}^{n}\left|A_{k}\right|^{2} \leq|t+1|^{2}+\sum_{k=1}^{n}\left|B_{k}\right|^{2}
$$

where $A_{k}=(k+1)\left(\frac{b_{k+1}}{b_{1}}-a_{k+1}\right) ; B_{k}=(k+1)\left(\frac{b_{k+1}}{b_{1}}+t a_{k+1}\right) ; a_{k}$ and $b_{k}$ are the coefficients of the functions $h(z)$ and $g(z) ; k=1,2,3, \ldots, n ; t=2 s-1 ; s=e^{-i \alpha} \cos \alpha$.

Proof Since

$$
g(z)=b_{1} z+b_{2} z^{2}+b_{3} z^{3}+\cdots \quad \Rightarrow \quad g^{\prime}(z)=b_{1}+2 b_{2} z+3 b_{3} z^{2}+\cdots
$$

We denote by $G(z)=\frac{1}{b_{1}} g(z)$

$$
\begin{aligned}
& G^{\prime}(z)=\frac{1}{b_{1}} g^{\prime}(z)=1+2 \frac{b_{2}}{b_{1}} z+3 \frac{b_{3}}{b_{1}} z^{2}+\cdots, \quad h(z)=z+a_{2} z^{2}+a_{3} z^{3}+\cdots, \\
& h^{\prime}(z)=1+2 a_{2} z+3 a_{3} z^{2}+\cdots,
\end{aligned}
$$

then we have

$$
\left\{\begin{array}{c}
\frac{1}{\cos \alpha}\left(e^{i \alpha} \frac{\frac{1}{b_{1}} g^{\prime}(z)}{h^{\prime}(z)}-i \sin \alpha\right)=p(z) \quad \Leftrightarrow \quad e^{i \alpha} \frac{\frac{1}{b_{1}} g^{\prime}(z)}{h^{\prime}(z)}=\cos \alpha p(z)+i \sin \alpha \\
\Leftrightarrow \quad \frac{\frac{1}{b_{1}} g^{\prime}(z)}{h^{\prime}(z)}=1+e^{-i \alpha} \cos \alpha(p(z)-1) .
\end{array}\right.
$$

Since $p(z)$ is in $P$, there is a function $\phi(z)$ satisfying the conditions of the Schwarz lemma such that

$$
p(z)=\frac{1+\phi(z)}{1-\phi(z)} \quad \Leftrightarrow \quad p(z)-1=\frac{2 \phi(z)}{1-\phi(z)}
$$

Using this equation in (2.12) and after the following calculations given above

$$
\frac{\frac{1}{b_{1}} g^{\prime}(z)}{h^{\prime}(z)}=1+e^{-i \alpha} \cos \alpha(p(z)-1)=1+s\left(\frac{2 \phi(z)}{1-\phi(z)}\right) \Rightarrow \text {, }
$$


we get the following equality:

$$
\frac{1}{b_{1}} g^{\prime}(z)-h^{\prime}(z)=\left(t h^{\prime}(z)+\frac{1}{b_{1}} g^{\prime}(z)\right) \text {. }
$$

If $\phi(z)=c_{1} z+c_{2} z^{2}+c_{3} z^{3}+\cdots$, we have

$$
\sum_{k=1}^{n} A_{k} z^{k}+\sum_{k=n+1}^{\infty} D_{k} z^{k}=\left[(1+t)+\sum_{k=1}^{n} B_{k} z^{k}\right] \phi(z)
$$

where

$$
\sum_{k=n+1}^{\infty} D_{k} z^{k}=\sum_{k=n+1}^{\infty} A_{k} z^{k}-\left(c_{1} B_{n} z^{n+1}+c_{1} B_{n+1} z^{n+2}+\cdots\right) .
$$

Therefore, the equality (2.15) can be considered in the following form:

$$
F(z)=G(z) \phi(z) .
$$

Using the Clunie method [6], then we can write

$$
\frac{1}{2 \pi} \int_{0}^{2 \pi}\left|F\left(r e^{i \theta}\right)\right|^{2} d \theta \leq \frac{1}{2 \pi} \int_{0}^{2 \pi}\left|G\left(r e^{i \theta}\right)\right|^{2} d \theta
$$

which gives

$$
\sum_{k=1}^{n}\left|A_{k}\right|^{2} r^{2 k}+\sum_{k=n+1}^{\infty}\left|D_{k}\right|^{2} r^{2 k} \leq\left(|t+1|^{2}+\sum_{k=1}^{n}\left|B_{k}\right|^{2} r^{2 k}\right)
$$

Eventually, we will let $r \rightarrow 1^{-}$, then we have

$$
\sum_{k=1}^{n}\left|A_{k}\right|^{2} \leq|t+1|^{2}+\sum_{k=1}^{n}\left|B_{k}\right|^{2}
$$

\section{Competing interests}

The authors declare that they have no competing interests.

\section{Authors' contributions}

All authors contributed equally and significantly to writing this paper. All authors read and approved the final manuscript.

\section{Author details}

${ }^{1}$ Department of Mathematics, Işık University, Meşrutiyet Koyu, Şile, İstanbul, Turkey. ${ }^{2}$ Department of Mathematics and Computer Sciences, İstanbul Kültür University, İstanbul, Turkey. ${ }^{3}$ Department of Mathematics, İstanbul Ticaret University, istanbul, Turkey.

\section{Received: 15 June 2012 Accepted: 24 September 2012 Published: 6 November 2012}

\section{References}

1. Duren, P: Harmonic Mapping in the Plane. Cambridge Press, Cambridge (2004)

2. Lewy, $\mathrm{H}$ : On the non-vanishing of the Jacobian in certain in one-to-one mappings. Bull. Am. Math. Soc. 42, 689-692 (1936)

3. Goodman, AW: Univalent Functions, vol. I. Mariner Publishing Company, Tampa (1983)

4. Goodman, AW: Univalent Functions, vol. II. Mariner Publishing Company, Tampa (1983)

5. Bernardi, SD: Convex and starlike univalent functions. Trans. Am. Math. Soc. 135, 429-446 (1969)

6. Clunie, J: On meromorphic Schlicht functions. J. Lond. Math. Soc. 34, 215-216 (1959) 
doi:10.1186/1029-242X-2012-262

Cite this article as: Aydog̃an et al.: Harmonic function for which the second dilatation is $\alpha$-spiral. Journal of Inequalities and Applications 2012 2012:262.

Submit your manuscript to a SpringerOpen ${ }^{\circ}$ journal and benefit from:

- Convenient online submission

- Rigorous peer review

- Immediate publication on acceptance

Open access: articles freely available online

- High visibility within the field

- Retaining the copyright to your article

Submit your next manuscript at $\boldsymbol{s p r i n g e r o p e n . c o m ~}$ 